Erikson, D. (1955). J. gen. Microbiol. 13, 136-148

\title{
Loss of Aerial Mycelium and other Changes in Strepto- mycete Development due to Physical Variations of Cultural Conditions
}

\author{
BY DAGNY ERIKSON \\ Bacteriology Department, University of Aberdeen
}

\begin{abstract}
SUMMARY: Several isolates of monosporous lines of Streptomyces coelicolor, both normally pigmented and colourless variants, were obtained by microdissection of young vegetative mycelia. In each case their properties largely resembled those of the parents. This material was studied together with three strains of Streptomyces scabies of varying degrees of virulence, which had been isolated from potato scab lesions. Modifications of growth were noted with respect to gross colonial morphology on agars containing detergents, structure of aerial mycelium arising from various types of surfaces, and development of germinating spores in liquids and in contact with non-nutritive plane surfaces. In both species-groups those organisms which produced least aerial mycelium on stock nutrient agar were most susceptible to the inhibitory influence of $0.01 \%$ Gemex 29 on the development of aerial mycelium in simpler media. Distorted aerial growths on detergent agar resembled irregular swollen sporogenous filaments produced in pectin + ammonium salt liquid media. These were also reminiscent of cases of sporogenesis in submerged cultures described by other workers. Apart from surface tensions operating at the air/medium interface, the relative degree of humidity in the culture vessel was found to have some effect upon variation of spore size. Germinating spores and young filaments growing in contact with plane surfaces were more prone to elongation than those growing in liquids. Branching was also influenced to a considerable extent by the nature of the physical environment.
\end{abstract}

In a recent review Hendlin (1954) pointed out that inadequate attention has in the past been paid to the profound effect which the physical environment must have upon the nutrition of micro-organisms. Such a viewpoint, which was restricted by Hendlin mainly to a consideration of such physical factors as temperature, $\mathrm{pH}$ values, oxidation-reduction potential, and $\mathrm{CO}_{2}$ tensions in so far as they cause perceptible nutritional differences, can almost certainly be extended to other aspects of microbiology. Thus the alteration of the energy relationships at interfaces which is brought about by the addition of surfaceactive agents to the media in which various acid-fast bacteria are grown is a recognized method of altering the character of the growth of the organisms (Alexander \& Soltys, 1946). Increased cell division and smaller cells result from the incorporation of Tween 80 in the media in which the non-acid-fast Nocardia turbata (Erikson, 1954) is cultivated. A certain degree of correlation has been noted between decreased humidity on the one hand, and increased production of sporogenous aerial mycelium by streptomycetes on the other (von Plotho, 1940; Erikson, 1947), suggesting that important phases in the development of a complex organism may be favoured or completely suppressed by fluctuations in the physical environment. 
In the course of regular platings of monosporous lines of an agar-digesting strain of Streptomyces coelicolor on a carbohydrate-free Czapek mineral salt agar medium, it was noted that the frequency of non-pigmented or asporogenous sectors was greatly increased by the addition of small quantities of non-toxic surface-active agents. It was further found that some of the changes in aerial mycelium brought about in this way resembled those occurring in liquid cultures containing pectin as a source of carbon. The present work is a comparative study of the differing details of growth shown by representatives of the species-groups $S$. coelicolor and $S$. scabies in a pectin +ammonium salt liquid medium and aqueous Czapek nitrate medium containing insoluble chitin; on plane and non-nutritive surfaces such as glass coverslips and cellophan films superimposed on different nutrients; and in agar media with and without the anionic detergent Gemex 29.

\section{ORGANISMS AND METHODS}

The representatives of the two common species-groups which were used were as follows.

Streptomyces coelicolor. Strain A3 (2): descendant of a single spore isolate (Erikson, 1948), possessing characteristic agar softening and pigment producing properties and bearing spirally coiled aerial sporophores. Strains A3 (2)/1-15: the progeny of 15 mycelial fragments dissected by means of a micromanipulator from a $24 \mathrm{hr}$. vegetative microcolony of A3 (2); all possessed apparently similar properties to the parent. Strain $12 \mathrm{~b}$ : a colourless variant, possessing little or no power of softening agar, having more or less straight aerial sporophores. Strains $12 \mathrm{~b} / 1-36$ : the progeny of 36 mycelial fragments dissected by means of a micromanipulator from a $\mathbf{2 4} \mathrm{hr}$. vegetative microcolony of $12 \mathrm{~b}$; all showed properties similar to the parent.

Streptomyces scabies. Strain Sc10: highly virulent to potatoes, producing a dark brown soluble pigment in protein media; strain Sc5: moderately virulent, also pigment producing; strain Sc4: not producing pigment in protein media but occasionally in pectin, weakly virulent. Strain Sc4: the most prolific in its production of a colourless to grey aerial mycelium on most substrates, but all strains capable of aerial growth, the individual sporophores being wavy to coiled. All these strains were isolated from potato scab lesions by $\mathrm{Mr}$ W. McKee of the Potato Storage Research Station, Loughborough, Leics., and kindly sent by him to me. The $S$. scabies strains were received in the freeze-dried state, and not opened until used for this study. The $S$. coelicolor strains were stored for 4 years in sterile soil after being prepared by micromanipulative methods as described.

The following media were employed:

Liquid media. A basal medium of Czapek mineral salts containing sodium nitrate with insoluble chitin (British Drug Houses Ltd.) as carbon source. This would not cause any alteration of surface tension even on prolonged incubation; or with the addition of $1 \%(w / v)$ of soluble starch or a sugar, e.g. sucrose, glucose. A pectin medium containing citrus pectin (Edward 
Gurr) and sodium ammonium phosphate prepared according to the method of Oxford (1944).

Solid media. The basal Czapek mineral salt medium containing $1 \%$ sucrose was solidified with $2 \%$ agar and combined with different sources of nitrogen: sodium nitrate, ammonium phosphate, sodium ammonium phosphate (all $0 \cdot 1 \%$ ), or urea $(0.5 \%)$. When plating with these media duplicates were poured, and to one set was added $0.01 \%$ of the detergent Gemex 29 (General Metallurgical and Chemical Industrial Ltd., London), which had been sterilized separately. Stock sporing growths used for inoculating the various types of cultures were maintained on 7-10-day slopes of starch $(1 \%)+$ casein hydrolysate $(0 \cdot 1 \%)$ agar medium.

Slide cultures. These were prepared according to the methods described for the germination of spores of Micromonospora vulgaris (Erikson, 1955a). Spores of the strains being studied were seeded directly on to the agar surface or on to superimposed cellophan films, both with and without the addition of coverslips. Various media described above were used together with the stock nutrient (peptone + Lab Lemco + Marmite) agar.

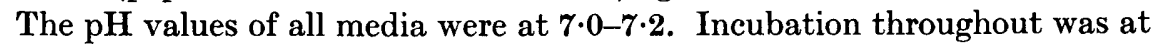
room temperature $\left(19-23^{\circ}\right)$. Observations were made by bright field or phasecontrast microscopy at daily or frequent intervals according to the progress of the growths.

\section{RESULTS}

\section{Gross colonial morphology in sucrose + mineral salt agar medium incorporating Gemex}

With urea as source of nitrogen. A representative series of colony-types has been selected from the large variety produced, and can be classified as those completely devoid of aerial mycelium and those where a slight aerial growth occurs centrally, peripherally, or in sectors. Of the Streptomyces scabies strains Sc 10 most frequently exhibited a smooth piled up aspect; strain Sc 5 a coherent skin-like growth with small colonies at the periphery, all entirely devoid of aerial mycelium, or with some slight marginal aerial mycelium; while strain Sc4 was the most consistent in producing a few sectors of aerial growth. Streptomyces coelicolor A3 (2) and all other isolates producing blue pigment grew abundantly to form a heavy corrugated membrane which lacked pigment and aerial mycelium. The colourless strains 12b/1-36 were least affected and grew moderately with a fair covering of aerial mycelium. In the control plates without Gemex growth was good throughout the series, and all colonies were evenly covered with their specifically typical aerial mycelium. When sucrose was omitted, there was poor growth in all cases except A3 (2) which showed the customary depressions round the colonies together with a normal development of aerial mycelium.

With sodium nitrate, ammonium phosphate, or sodium ammonium phosphate as source of nitrogen. The relative susceptibilities of the different strains to the action of Gemex remained much the same, although the amount of growth produced varied proportionately according to the nitrogen source-most 
abundant with sodium ammonium phosphate and least with sodium nitrate. Throughout the series the Streptomyces scabies strains-Sc10, Sc5, Sc4-in that order showed the most marked tendency towards complete or partial loss of aerial mycelium. The Streptomyces coelicolor A3 (2) pigmented strains on the whole retained their characters better, but in any one plate variable aspects could generally be found. Such growths were more evident in nitrate nitrogen plates. The colourless strains $12 \mathrm{~b} / 1-36$ produced the most consistently uniform colonies. In the control series without Gemex good characteristic growth was obtained with all. The agar digesting A3 (2) strain was the only one to grow equally well without sucrose.

\section{The structure of aerial mycelium arising from different surfaces}

(a) From agar media as above. Spiral sporophores, either tightly coiled (Streptomyces coelicolor A3 (2): Pl. 1, fig. 4) or of varying degrees of looseness (S. scabies Sc 10: Pl. 1, fig. 1) were normally characteristic of the majority of the strains. Exceptions were the little more than wavy and often straight sporophores of the $12 \mathrm{~b} / 1-36$ isolates. No noteworthy differences were found between the urea and mineral salt media. The addition of Gemex, where it resulted in a partial development of aerial mycelium, also tended towards a straightening of the sporophores or sterility, or both. In those instances where the gross aspect seemed unchanged, as in the relatively good development of aerial mycelium by the isolates $12 \mathrm{~b} / 1-36$, a more detailed examination revealed considerable distortion of the sporogenous filaments. The most noticeable feature of this type of aerial growth was the thickening of many filaments, which were often swollen so as to form clavate tips.

(b) From aqueous mineral salt or pectin media. The clearest differences were observed in the 12b/1-36 isolates, whose vigorous ability to produce aerial mycelium in most circumstances has already been noted. Sporophores typical of this group arising by simple monopodial branching from a filament of the vegetative mycelium produced relatively long chains of spores. When grown at the surface of a pectin +ammonium salt medium (Pl. 1, fig. 2), the whole filament and its short lateral branches were sporogenous and resembled the submerged sporing growths pictured by Carvajal (1946) and by Pénau, Hagemann, Velu \& Peyre (1954) for Streptomyces griseus. It will be noted also that there were many swollen, large, and clearly empty cells. In many respects this type of sporulating growth showed considerable similarity to that produced on the detergent agar media (Pl. 1, fig. 5).

(c) From cellophan over agar media. Growth on cellophan is necessarily thinner and more evenly spread over the film as the result of the slow diffusion of nutrients. Hence it is easier to observe the progressive stages of development on such films than in the more compact cell complexes produced in direct contact with agar, or partially immersed in liquid media. The Streptomyces coelicolor A3 (2) strains which required no carbohydrate added, when grown on cellophan over a mineral salt medium containing only nitrate, 
spread so evenly over the film (Pl. 1, fig. 3) that the regularity of both vegetative and sporogenous aerial filaments was clearly visible. The well-spaced growths on films over such simple media permitted observation of the way in which the aerial filaments arise by monopodial branching from any vegetative filament anywhere over the film. All the strains examined grew in this way, but with the addition of more nutrients to the agar the readier accessibility of the food supply allowed greater scope to the inherent tendency of actinomycetes to grow in a circumscribed fashion, i.e. to extend their cell-masses upwards by means of intertwined and branching filaments, instead of individually outwards in the manner of fungi. Thus with richer growths the origins of single aerial filaments could as a rule be discerned only at the margins. In no instances could any evidence be obtained of fusion of one or more filamentous cells to yield an 'initial cell' from which the secondary aerial growth was derived (Klieneberger-Nobel, 1947; McGregor, 1954).

\section{Qualitative and quantitative differences in spore production}

(a) On agar media with and without Gemex. The distortion of the sporogenous filaments sometimes produced on Gemex-containing media (PI. 1, fig. 5) frequently resulted in a diminution in the spore yield. Comparison of impression preparations of the same strain (12b/36) grown on sucrose ammonium phosphate agar for the same length of time (2 weeks) with (Pl. 1, fig. 5), and without (Pl. 1, fig. 9) Gemex, will show this clearly. The total mass of cell material synthesized in the upper stratum of growth arising from detergent agar was at least as great as the normal growth in the control plate, but many of the swollen filaments failed to produce spores (Pl. 1, fig. 5). Such spores as were formed were usually in short chains, relatively few in numbers. The clavate terminal cells, as well as other irregularly shaped filamentous cells, were viable and germinated in the ordinary way under suitable circumstances. Nevertheless, the quantitatively higher yield of spores from a similar amount of filamentous growth in the control (Pl. 1, fig. 9) seemed a more economical use of the cell material from the point of view of distribution.

In the Streptomyces coelicolor species-group the typical pigmented A3 (2) strains were devoid of aerial growth on detergent media in most instances, and displayed a very regular development of spores throughout the spiral twists of the normal sporophores produced on ordinary media. In older colonies, however, long aerial strands in the form of interconnecting overhead bridges were often found to extend to adjacent colonies (Pl. 1, fig. 4), and from these might develop subsequent overgrowths of colourless more or less straight aerial strands. Such overgrowths, which probably obtained their nutrient syntrophically from the disintegrating filaments below, frequently showed inequalities in size on the part of the spores in long chains (cf. Pl. 1, fig. 9).

Sampling the spores by means of impression preparations clearly yielded information only concerning the superficial layer of growth, which was furthest removed from the source of food supply, and which might therefore 
be readily subject to slight variations. The random samplings taken from spore suspensions, especially those prepared from slopes in stoppered bottles, generally revealed a more uniform appearance ( $\mathrm{Pl} .1$, fig. 8). It is of interest to compare these relatively large round spores produced in humid conditions with those which have fallen naturally from the crown of a colony to its perimeter on the thin film of drying agar in a slide culture (Pl. 1, fig. 6). The latter are slenderer, more oblong, and more varied in appearance, yet both specimens were from the same strain $(12 \mathrm{~b} / 7$ grown for the same time on the same medium (8 days on Czapek sucrose nitrate agar)). It was frequently found that differences in the depth and consistency of the agar, and especially in the degree of humidity obtaining in the culture vessel, resulted in variations both as to total numbers and aspect of the spores produced.

(b) On liquid media. In unshaken, unaerated cultures of simple aqueous Czapek media containing only dilute solutions of sugars or insoluble chitin or cellulose in the form of filter-paper strips, the thin network of surface filaments commonly gave rise to simple aerial sporophores typical of the respective strains. Shallow layers of such liquids in flasks or bottles or in Petri dishes supported by circles of filter-paper or cellophan permitted the development of more coherent superficial growth than in test-tubes, and small portions could readily be removed for microscopical examination without undue disturbance of the essential relationships of the growth. In such systems the yield of spores was determined by the area occupied by the medium and not by its volume; bottom growth was consistently vegetative only. Long chains of spores were developed in cultures of Streptomyces scabies and $S$. coelicolor (Pl. 1, fig. 7), but tended to fragment very readily. Spore size throughout was fairly constant, according to the species and strain. Spore chains were found, derived from cultures on both liquid (Pl. 1, fig. 7) and solid media, in which the constituent spores appeared to branch laterally. On closer examination these lateral spores were often found to arise from the isthmus between two spores in a chain (cf. Carvajal, 1946).

As already mentioned, the more plentiful but partially submerged growths in pectin media was usually characterized by an increase in the variety of shapes and sizes of spores together with a decrease in numbers. In the Streptomyces scabies Sc4 strain growth was less distorted and the spores more uniform. On the whole, the most significant feature in the pectin growths was the frequent occurrence of thickened filaments, clubs and clavate terminal cells (Pl. 1, fig. 2). These structures resembled very closely the 'massues' and 'éléments spiciformes' described by Pénau et al. (1954) in their submerged cultures of $S$. griseus, and also on the pectin-coated coverslips partially immersed in a rich nutrient broth from whose examination they made their deductions concerning surface growth. The broth employed by these workers contained peptone, meat extract and glucose, and would therefore have a surface tension lower than that of watery solutions of mineral salts (see Glassman, 1948). 


\section{Germination of spores}

(a) In liquids. Where there was no impediment to growth in any plane, e.g. in a tube of aqueous Czapek sucrose medium, spores rarely exhibited any change in shape prior to germination, and produced as a rule one short germtube which quickly branched in different directions (Pl. 2, fig. 10). The mycelium developing naturally in these circumstances soon became the typical streptomycete colony composed of branching filaments intertwining in every possible way, into whose interior visual inspection became increasingly difficult (Pl. 2, fig. 11).

(b) On cover-slips receiving a fluctuating supply of liquid nutrients. When a spore suspension in broth was run along the undersurface of a coverslip inclined at an angle over agar strips forming an enclosed chamber, spores adhering to the glass showed varying degrees of elongation (cf. Pl. 2, figs. 13-17) and might develop one to three germ-tubes. In such a system the supply of nutrients was locally restricted, with the result that 'scrolls' of elongated filaments following the movements of moisture on the glass were often formed (cf. similar development on cellophan, Pl. 2, fig. 12). Occasionally also, especially with the Streptomyces scabies strains which were characterized by a more wavy growth on the part of the vegetative filaments than $S$. coelicolor (cf. Pl. 2, fig. 12, with Pl. 1, fig. 3) spores germinating on the glass surface developed 'loops' or 'nests' of filaments (Klieneberger-Nobel, 1947; 'vrilles' and 'nids', Pénau et al. 1954). Such structures developed away from the glass as the growing filaments shifted direction slightly in accordance with the varying food supply. A photograph of such a 'loop' produced under identical conditions to those described has already been published for Micromonospora vulgaris (Erikson, 1953: pl. 1, fig. 6). Yet, as can be seen by the more recent illustrations of germinating spores of $M$. vulgaris (Erikson, 1955a: pl. 2, figs. $7-10$; pl. 3, figs. 11, 16; pl. 4, figs. 17, 18), this particular thermophilic actinomycete normally produced long straight, or slightly wavy, loosely branching filaments on agar surfaces or in complete immersion in liquid cultures.

(c) On moist cellophan films over agar media. Germination on moistened membranes through which an adequate supply of nutrients diffused evenly appeared little different from the same process observed directly on a moistened agar surface (see Pl. 2, figs. 13-17). The most noteworthy feature of young growths on cellophan was the way in which the growing filaments extended for relatively long distances over the film, branching and developing during the early stages only within the plane parallel to the film (Pl. 2, fig. 12). If the spores were well spaced, it was then always possible to note the way in which advancing growing tips recoiled, crossed over or under, or extended in parallel strands with other filaments of the same or adjacent growths. Several clear instances of this lack of anastomosis can be seen in the present illustration (Pl. 2, fig. 12), as well as in that of a similar cellophan growth of the thermoduric strain Streptomyces albus 1569 accompanying another article (Erikson, 1955 b: Pl. 2, fig. 14). All actinomycetes studied by these techniques have 
revealed the same manner of growth. As Pénau et al. (1954) have pointed out with regard to specimens of submerged growths of $\boldsymbol{S}$. griseus artefacts can readily give rise to the appearance of hyphal fusion but when the living cells are examined by phase-contrast microscopy the enlarged 'fusion cell' is usually resolved into the projection of one filament crossing over another (see Pénau et al. 1954: pl. 1, fig. 7).

(d) Between coverslip and agar surface. When individual spores of Streptomyces coelicolor in slide cultures were examined and photographed at intervals, there was a more perceptible elongation of the germinating spore ( $\mathrm{Pl} .2$, fig. 13: top right-hand spore) which might be partly attributable to the compression of the coverslip (cf. germinating spore in liquid, Pl. 2, fig. 10). It was also noted that a greater initial degree of elongation was apparent when a spore produced two germ-tubes (Pl. 2, fig. 14: same top right-hand spore $12 \mathrm{hr}$. later) in comparison with a spore producing first one germ-tube (Pl. 2, fig. 14: bottom left-hand spore) and then another after an interval (Pl. 2, fig. 15: the same bottom left-hand spore $18 \mathrm{hr}$. later). At this stage when both spores had produced two germ-tubes, it became obvious that the difference in size evident in Pl. 2, fig. 13, was a real distinction and not just a result of the earlier germination on the part of the one spore. A little later (Pl. 2, fig. 16: after $\mathbf{2 4} \mathrm{hr}$.) it was seen that the larger spore had synthesized a greater amount of cell material in the shape of two twisting filaments. At this stage burrowing into the agar had started and the complete growth was no longer in focus.

In another field of the same slide culture (Pl. 2, fig. 17: 3 days after sowing) four spores were found to have developed in slightly different ways. The one at the extreme right possessed three germ-tubes and showed a moderate degree of elongation, while a very similar appearance was given to the spore in the top left-hand as the result of the emergence in two germ-tubes. On the other hand, the one immediately beneath it was foreshortened as if the long axis-if indeed it were not almost spherical—had been turned downwards into the agar. This spore had only one germ-tube. Very little elongation, if any, was shown by the fourth spore to the left centre, which had a main short germ-tube from which two long branches had developed, as well as a second subsidiary germtube above the small sterile spore trapped between it and the main right-hand branch.

Throughout all the studies on germination it was found that living streptomycete spores appeared as homogeneous dense bodies when viewed by phasecontrast microscopy, whatever the methods of cultivation. Since they were not refractile like the spores of the thermophilic Micromonospora vulgaris, there could be no perceptible changes in density prior to germination. Those spores that occasionally appeared clear and empty, particularly in pectin or Gemex preparations, constantly proved non-viable. The density of the spores was such as to preclude the appearance during life of any internal structures. 


\section{DISCUSSION}

The ability to produce an aerial mycelium, so characteristic a property of the genus Streptomyces, implies the capacity on the part of a large number of cells to pass beyond the surface boundary of the nutrient phase, whether solid or liquid, and then to obtain nourishment both for their own maintenance and for the synthesis of fresh cell material by means of an internal pipeline from the basal source of supply. This is a fundamentally different situation from that obtaining in the typical eubacterial culture where all but the superficial cells, and even they over at least half their area, can absorb individually through their external membranes the dissolved solutes requisite for growth. When this is borne in mind, it is logical to expect that local variations in the physical state of the substratum upon which a given streptomycete culture grows might readily affect the ability of certain cells to grow away from the medium/air interface. Moreover, even when such filamentous extension away from the nutrient source has taken place, slight differences in tension in the immediate environment of the basal cells may selectively alter or completely interrupt the transportation of soluble substances to the apical portion of the aerial growth.

It has been shown that the lowering of the surface tension of agar media by means of a non-toxic detergent resulted in inhibition or partial loss of aerial growth in most of the strains studied. The organisms which commonly produced the least aerial mycelium and rarely any at all on nutrient agar, such as the most virulent Streptomyces scabies strain Sc 10, were the most susceptible to the inhibitory influence; while in the same group the least virulent strain Sc4 showed similar powers of aerial mycelium production on nutrient and detergent agars. On the other hand, the characteristic blue-pigmented A3 (2) strains of $S$. coelicolor produced normally both aerial sporogenous and vegetative phases on simple media containing inorganic nitrogen sources, but only the vegetative phase on rich complex media like nutrient agar. It is these strains which were most responsive to the inhibitory action of artificially lowered surface tension as regards aerial mycelium production in detergent media, whereas the colourless $12 \mathrm{~b}$ strains were able to develop a moderate amount of aerial growth on both nutrient and detergent agars.

Correlation of paucity or complete suppression of aerial growth in both nutrient and detergent agars is of significant interest, since culture media containing protein ordinarily have surface tension values lower than that of water (Glassman, 1948). A careful scrutiny of the descriptions compiled by Waksman (1953) of a large number of known species of Streptomyces will reveal that in sixty-eight instances $(\mathbf{4 6} \cdot 5 \%$ ) absence or very scanty development of aerial mycelium on nutrient agar was reported as compared with moderate or good growth on a defined medium with a simpler nitrogenous source. In the large majority of these instances moderate to good, if not luxuriant, vegetative growth was produced, indicating that the organisms experienced little difficulty in metabolizing the complex media. It would therefore seem probable that it was the physical rather than the chemical 
components of the system that were active in preventing aerial growth. An analogous example can be observed in the potency of the physical factor of gas phase in connexion with the development of aerial mycelium by the thermophilic actinomycete Micromonospora vulgaris: aerial mycelium in atmospheric air but not in oxygen (Webley, 1954).

The distorted, swollen, and often empty cells found in the sporogenous secondary mycelium of those organisms which did produce aerial growth on detergent media resembled very closely the cells arising in abnormally short filaments from the surface and subsurface of pectin media. Again it would seem that the operative factor common to both systems is the physical one of surface tension. Both types of culture show examples of cytolysis such as often result from the contact of surface active agents with biological cells. Moreover, they show a very strong resemblance to the thickened rods, swollen buds, enlarged clavate tips and clubs described and illustrated by Pénau et al. (1954) for Streptomyces griseus when grown on pectin-coated coverslips in rich complex nutrient broth. The medium used by these authors would certainly have a surface tension value lower than that of water, even without the addition of dissolved pectin, while its richness would ensure the abundant production of susceptible cells.

Bloated shapes resulting from over-feeding and other unfavourable conditions are of common occurrence in many varieties of actinomycetes (cf. the large clubs and hexagonal cells of Nocardia sebivorans growing on the surface of solid paraffin: Erikson, $1955 b$, pl. 1, fig. 8). As Villemin, Lechevalier \& Waksman (1953) have pointed out, such gross overfeeding as is normally involved in the cultivation of actinomycetes for antibiotic formation can be regarded as constituting 'pathologic' conditions in comparison with the austerity of the fare obtainable in the natural habitat-the soil. It is in such over-rich artificial environments, complicated in the case of submerged aerated cultures by the additional physical factor of vibration, that the variety of cell elements described by Pénau et al. (1954) has been found. It must also be remembered that Streptomyces griseus, the organism with which they worked, is extremely variable even for a streptomycete (see Waksman, Reilly \& Harris, 1948).

No clear cases of anastomosis were found by Pénau et al. (1954), confirming the observations of Erikson (1949). This is of importance genetically, if we accept Whitehead's (1948) thesis that it is more important for a proposition to be interesting than to be true, for the most interesting theory concerning the origin of the aerial mycelium depends on the assumption of hyphal fusion. Klieneberger-Nobel (1947) did not observe any clear cases of fusion, and merely suggested the possibility of such occurrences within the tangled 'nests' of filaments to be found in partially immersed coverslip growths. A criticism of this technique and of the physical factors involved in such growths has already been made (see earlier section: Germination of Spores $(b)$ ). Examination of well-spaced growths on poor nutrient media (see Pl. 1, fig. 3) will constantly reveal simple monopodial branching on the part of the aerial filaments, without the intervention of any enlarged 'initial cell', just as it has been described 
by all the earlier workers (see Ørskov, 1923). Moreover, wild streptomycetes in soil show the same characteristic mode of growth when developing on buried coverslips (see Erikson, 1947: pl. 1, fig. 2). Yet Bisset (1950) has raised Klieneberger-Nobel's theory to the status of dogma without the benefit of further confirmatory evidence or reference to earlier contradictory data. Some of the drawbacks to the methods employed by Bisset in elucidating cytological findings have been pointed out by DeLamater (1954).

The extreme plasticity of the actinomycetes in general in response to environmental conditions is well known to those who have long occupied themselves with these organisms, but is perhaps less vividly realized by those mainly preoccupied with eubacteria. It is generally the latter workers who have attempted to find significance in unusually shaped cells produced in special circumstances. On the other hand, Gottlieb (1953) has stressed the heterogeneity of streptomycete cultures as a result of observing that growth is confined almost entirely to the apical region of the hypha, in this respect resembling the fungi. In my experience it is this fact-that in mature growths in complex media large portions of the vegetative mycelium are dead or dying, although still permitting the passage of nutrients to the living cells, that accounts to a large extent for the delimitation of the vital protoplasm within thickened filaments, enlarged 'clubs', 'éléments spiciformes', 'segmentation spores', etc. Such structures may persist through adverse circumstances and germinate to form a typical mycelium in suitable environments, but they cannot therefore be regarded as reproductive organs.

In the present study different strains of Streptomyces scabies reacted differently to surface tension factors in producing little or no aerial mycelium on detergent agars, and showed varying degrees of susceptibility to other changes of the physical environment. The pigmented A3 (2) and the colourless $12 \mathrm{~b}$ variants of $S$. coelicolor also responded in varying ways. These two forms were originally obtained from single spores. On the other hand, the various isolates of A3 (2) which were obtained as the result of micromanipulative dissection of a very young homogeneous vegetative mycelium behaved throughout very similarly. This was the case also with the $\mathbf{1 2} \mathbf{b}$ isolates of similar origin. Such results uphold the conclusions drawn from earlier work carried out in less detail (Erikson, 1948).

This work was done by the author as a member of the scientific staff of the Agricultural Research Council. I wish to thank Professor J. Cruickshank, C.B.E., for the hospitality of his department; Miss L. M. Crump and Dr H. G. Thornton, F.R.S., of Rothamsted Experimental Station for help with the original micromanipulations; and Miss Mildred Mackay for technical assistance.

\section{REFERENCES}

Alexander, A. E. \& Soltys, M. A. (1946). The influence of surface active substances on the growth of acid-fast bacteria. J. Path. Bact. 58, 37.

Bisset, K. A. (1950). The Cytology and Life-History of Bacteria. Edinburgh: Livingstone. 
Carvajal, F. (1946). Studies on the structure of Streptomyces griseus. Mycologia, $38,587$.

Delamater, E. D. (1954). Cytology of Bacteria. Part II. The bacterial nucleus. Annu. Rev. Microbiol. 8, 23.

Erikson, D. (1947). Differentiation of the vegetative and sporogenous phases of the actinomycetes. II. Factors affecting the development of the aerial mycelium. J. gen. Microbiol. 1, 45.

ERIKSON, D. (1948). Differentiation of the vegetative and sporogenous phases of the actinomycetes. III. Variation in the Actinomyces coelicolor species-group. J.gen. Microbiol. 2, 252.

Erikson, D. (1949). The morphology, cytology, and taxonomy of the actinomycetes. Annu. Rev. Microbiol. 3, 23.

Erikson, D. (1953). The reproductive pattern of Micromonospora vulgaris. J. gen. Microbiol. 8, 449.

Erikson, D. (1954). Factors promoting cell division in a 'soft' mycelial type of Nocardia: Nocardia turbata n.sp. J. gen. Microbiol. 11, 198.

ERIKson, D. (1955a). Changes in refractility and permeability accompanying germination of heat-resistant spores of Micromonospora vulgaris. J. gen. Microbiol. 13, 119.

Erikson, D. $(\mathbf{1 9 5 5 b})$. Thermoduric properties of Nocardia sebivorans and other pathogenic aerobic actinomycetes. J. gen. Microbiol. 13, 127.

Glassman, H. N. (1948). Surface active agents and their application in bacteriology. Bact. Rev. 12, 105.

GotTlieb, D. (1953). The Physiology of the Actinomycetes. In Actinomycetales, Symp. VI Congr. int. Microbiol. p. 122.

Hendlin, D. (1954). The nutrition of microorganisms. Annu. Rev. Microbiol. 8, 47.

KLieneberger-Nobel, E. (1947). The life cycle of sporing Actinomyces as revealed by a study of their structure and septation. J. gen. Microbiol. 1, 22.

McGregor, J. F. (1954). Nuclear division and the life cycle in a Streptomyces sp. J. gen. Microbiol. 11, 52.

Ørskov, J. (1923). Investigations into the Morphology of the Ray Fungi. Copenhagen: Levin and Munksgaard.

Oxford, A. E. (1944). Production of a soluble pectinase in a simple medium by certain plant-pathogenic bacteria belonging to the genus Pseudomonas. Nature, Lond. 154, 271.

Pénau, H., Hagemann, G., Velu, H. \& Peyre, M. (1954). Modalités du cycle evolutif de Streptomyces griseus en culture profonde. Rev. Immunol. 18, 265.

Plotro, O. von (1940). Beiträge zur Kenntnis der Morphologie u. Physiologie der Actinomyceten. Arch. Mikrobiol. 11, 33.

Villemin, P. F., Lechevalier, H. A. \& Waksman, S. A. (1953). Antibiotics of Actinomycetes with Special Reference to their Role in the Physiology of the Organisms Producing them. Actinomycetales, p. 137. Symp. VI Congr. int. Microbiol.

Waksman, S. A. (1953). The Actinomycetes. Part I in Guide to the Classification and Identification of the Actinomycetes and their Antibiotics. Baltimore: Williams and Wilkins.

Waksman, S. A., Reilly, H. G. \& Harris, D. A. (1948). Streptomyces griseus (Krainsky) Waksman \& Henrici. J. Bact. 56, 254.

WEBLEy, D. M. (1954). The effect of oxygen on the growth and metabolism of the aerobic thermophilic actinomycete Micromonospora vulgaris. J. gen. Microbiol. 11, 114.

Whitehead, A. N. (1948). Adventures of Ideas, p. 280. Aylesbury and London: Pelican Books Ltd. 


\section{EXPLANATION OF PLATES}

Phase-contrast preparations.

Plate 1

Fig. 1. S. scabies, Sc 10, 8 days on sucrose urea agar, showing loosely coiled spiral sporophores. $(\times 720$. $)$

Fig. 2. S. coelicolor, $12 \mathrm{~b} / 10,24$ days at surface of pectin ammonium salt liquid medium, showing sporogenous filament with short lateral branches and many of the cells abnormally large or empty. $(\times \mathbf{2 6 4 0}$. $)$

Fig. 3. S. coelicolor, A3 (2), 4 days on cellophan over Czapek nitrate agar without added carbon source, showing loosely coiled aerial filaments arising by monopodial branching from the vegetative mycelium. $(\times 720$.

Fig. 4. S. coelicolor, A3 (2), 4 weeks on sucrose ammonium salt agar, showing sporophores from adjacent colonies intertwining by means of their tightly coiled spirals. $(\times 720$. $)$

Fig. 5. S. coelicolor, $12 \mathrm{~b} / 36,3$ weeks on sucrose ammonium salt Gemex agar. Impression preparation showing distorted sporogenous filaments. $(\times 2160$. $)$

Fig. 6. S. coelicolor, $12 \mathrm{~b} / 7,8$ days on sucrose nitrate agar slide culture. Slender, elongated spores fallen from crown of colony to perimeter; cf. Pl. 1, fig. 8 . $(\times \mathbf{2 1 6 0}$.)

Fig. 7. S. scabies, Sc 4, 8 days at surface of chitin mineral salt medium, showing chain of spores with apparent lateral branching. $(\times \mathbf{2 6 4 0}$. $)$

Fig. 8. S. coelicolor, 12b/7, 8 days on sucrose nitrate agar slope in stoppered bottle. Aqueous spore suspension from growth. $(\times \mathbf{2 1 6 0}$. $)$

Fig. 9. S. coelicolor, 12b/36, 2 weeks on sucrose ammonium salt agar. Impression preparation showing long mainly straight chains of spores, with some spores larger than the normal size; cf. fig. 5. $(\times 2160$.

\section{Plate 2}

Fig. 10. S. scabies, Sc 4, $48 \mathrm{hr}$. in sucrose Czapek liquid medium, showing germinating spore with very short germ tube giving rise to three branches. $(\times 2640$. $)$

Fig. 11. S. scabies, same as fig. 10, 4 days later, showing portion of closely intertwined mycelial growth. $(\times 800$. $)$

Fig. 12. S. scabies, Sc 5, 4 days on cellophan over sucrose ammonium salt agar, showing manner in which advancing filaments cross over one another or extend in parallel strands. $(\times 640$. $)$

Fig. 13. S. coelicolor, A3 (2), portion of slide culture on nutrient agar sealed with coverslip, showing two spores of different sizes prior to germination. $(\times \mathbf{4 3 2 0}$.)

Fig. 14. Same as fig. 13, $12 \mathrm{hr}$. later, showing two germ tubes produced by elongated top right-hand spore, and only one germ tube by the smaller spore. $(\times 4320$. $)$

Fig. 15. Same as fig. 13, $18 \mathrm{hr}$. later. The smaller spore has now produced a second germ tube. $(\times 4320$.

Fig. 16. Same as fig. 13, $24 \mathrm{hr}$. later, showing twisting of young filaments burrowing into agar with the result that growth is partly out of focus. $(\times 4320$.)

Fig. 17. Another field of the same slide culture as in fig. 13, 3 days after sowing, showing four germinated spores and one sterile. The number of germ tubes produced varies between 1 and 3 . $(\times 2800$. $)$

(Received 21 January 1955) 
Journal of General Microbiology, Vol. 13, No. 1
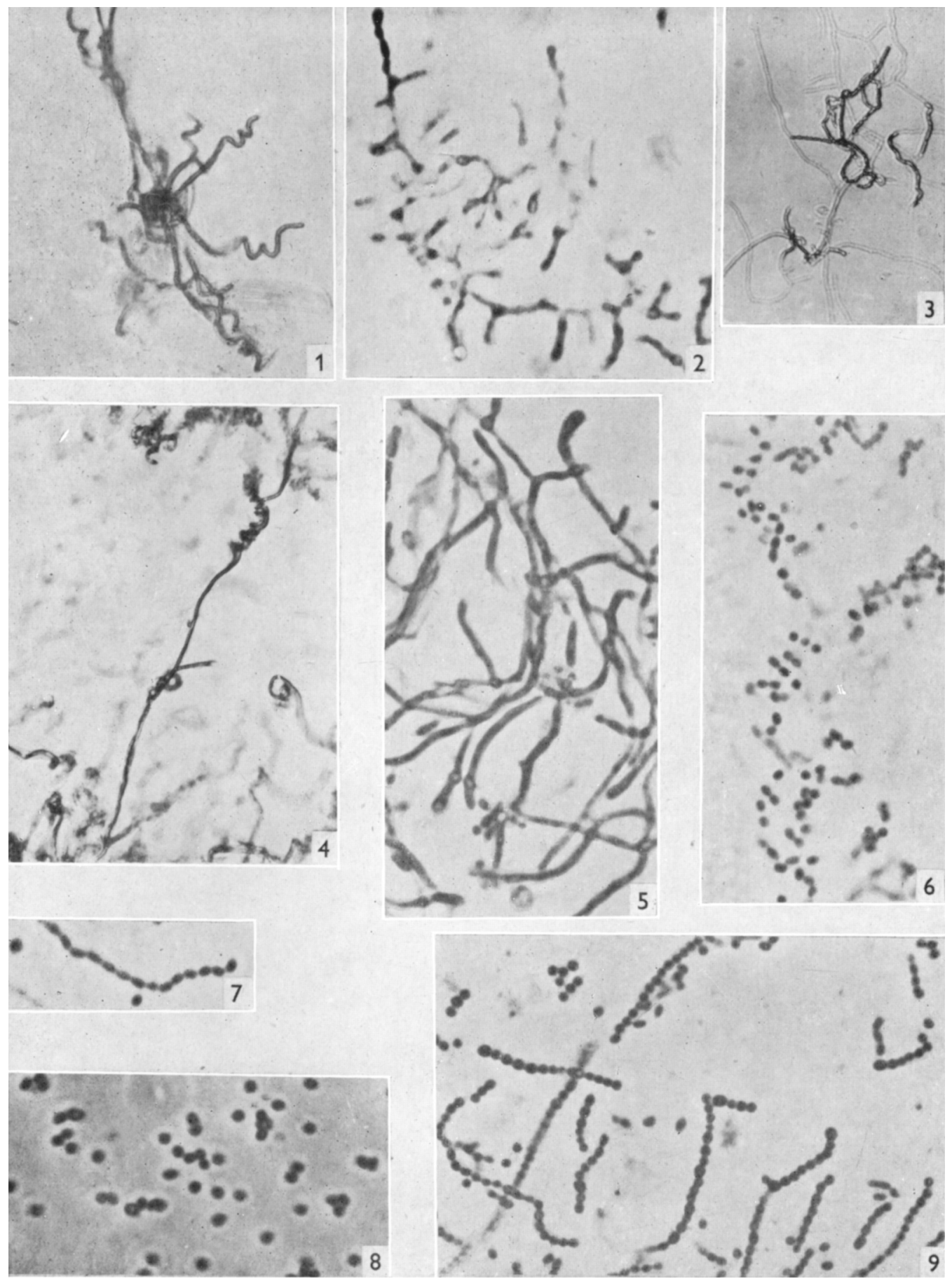

D. Erikson-physical factors affecting streptomycetes. Plate 1 
Journal of General Microbiology, Vol. 13, No. 1
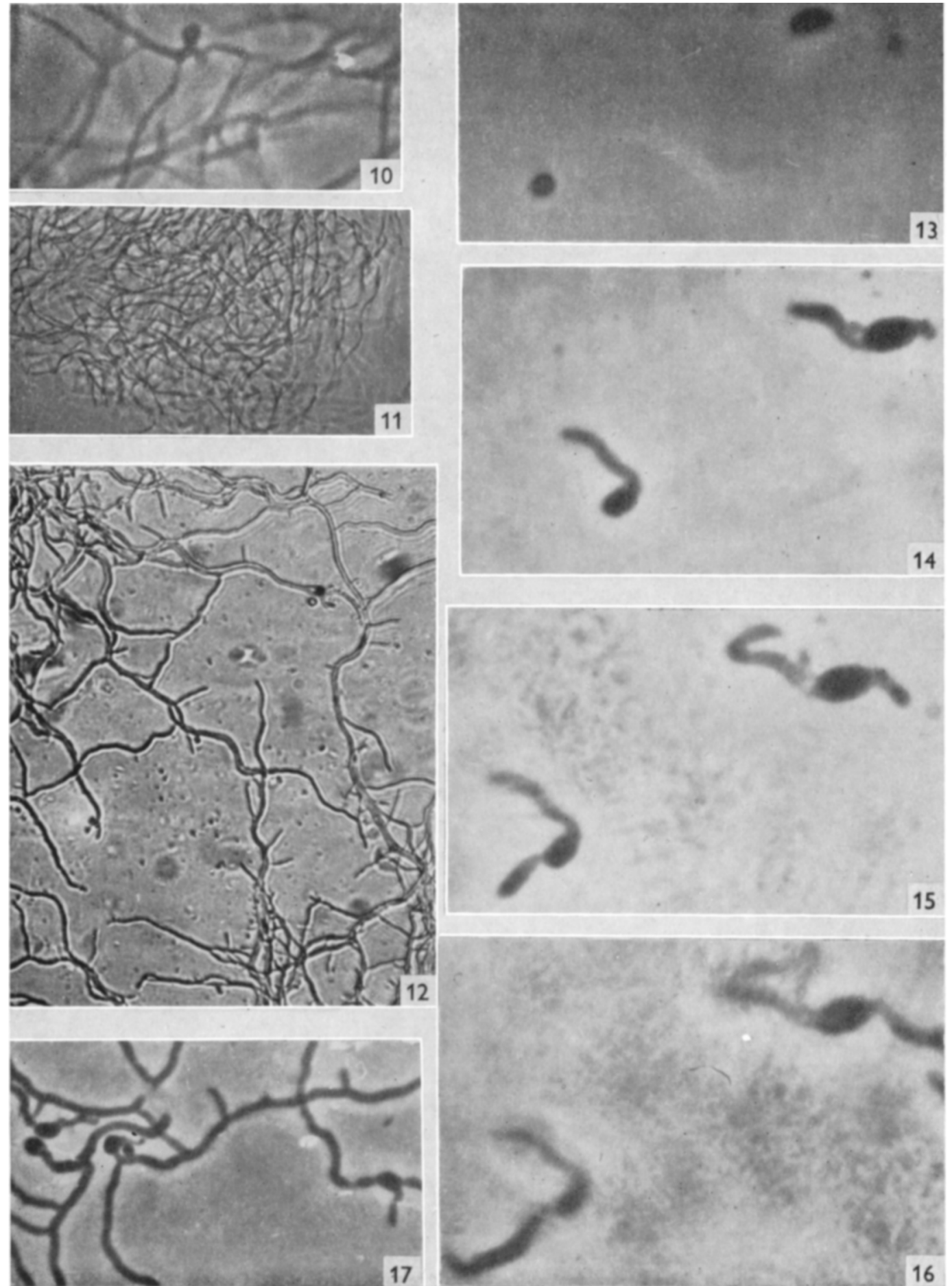

D. Erikson-Physical factors affecting streptomycetes. Plate 2 